\title{
Factors influencing medication adherence and hypertension management revisited: recent insights from cancer survivors
}

\author{
Manuel Morgado and Sandra Rolo \\ Hypertension Research (2012) 35, 894-896; doi:10.1038/hr.2012.100; published online 5 July 2012
}

$\mathrm{L}^{2+a}$ ack of adherence to antihypertensive $\mathrm{Cmedication}_{\mathrm{m}}$ a major reason for poor blood pressure (BP) control worldwide. Generally, adherence rates to long-term pharmacotherapies averages only from 50 to $65 \%$, regardless of the illness, regimen or measurement criteria. ${ }^{1}$ Poor adherence to long-term medications severely compromises the effectiveness of treatment, making this a crucial issue, both from the perspective of patient outcomes and quality of life and health economics. ${ }^{1}$

Non-adherence to BP-lowering medication may reduce treatment benefits, but can also distort assessment of the efficacy of treatment. ${ }^{2}$ If a physician is not aware of the fact that the hypertensive patient is not taking the drug as prescribed, he may attribute uncontrolled BP to ineffectiveness of the drug and may unnecessarily increase the dose, change or add another drug.

From a health economics standpoint, it is estimated that non-adherence to medication regimens may cost as much as $\$ 300$ billion to the United States of America healthcare system. $^{3}$ Ineffectiveness of treatment and disease progression due to non-adherence may lead to poor outcomes, disease complications, hospitalizations, emergency department visits and even death. ${ }^{3,4}$

Increased medication adherence for chronic conditions can have the effect of significantly keeping patients out of the

M Morgado and S Rolo are at the Pharmaceutica Services of Hospital Centre of Cova da Beira, Covilhã Portugal; M Morgado is also at the CICS-UBI - Health Sciences Research Centre, University of Beira Interior, Covilhã, Portugal.

E-mail: manuelaugustomorgado@gmail.com hospital, improving quality of life and cutting medical costs. The World Health Organization (WHO) stated that 'Increasing the effectiveness of adherence interventions may have a far greater impact on the health of the population than any improvement in specific medical treatments.

Researchers have identified several factors that may influence a patients' adherence to antihypertensive medication. The asymptomatic characteristic and lifelong nature of hypertension are two key factors that undoubtedly contribute to poor adherence to drug regimens. ${ }^{1}$ Several other factors affect medication adherence in hypertensive patients: ${ }^{5,6}$ (1) social and economic factors, such as social support and socioeconomic status (cost of medications); (2) factors related to the health care team/system, such as clear communication and time spent explaining the disease and the treatment; (3) therapy-related factors such as complexity of medication regimen (dosing frequency and number of medications) and side effects; and (4) patient-related factors such as emotional state, health beliefs and health literacy.

Interventions to improve medication adherence encompass self-management programs that include educational and behavioral strategies in the form of multidisciplinary care. Behavioral strategies use techniques such as reminders, reinforcement of the importance of medication adherence and home BP monitoring in hypertensives. Adapting medication regimens to the patients' needs is sometimes used by healthcare providers. This includes analysing patients' conveniences, simplifying drug regimens and using adherence tools. In many chronic diseases, interventions concentrate on patient education providing instructions about the treatment, reducing number of drugs and frequency of doses, providing education about expected adverse effects, and motivating patients to adhere to healthy lifestyle habits. Educational interventions have been found to be useful in improving knowledge about the seriousness of a disease and the importance of medication adherence. It is important to teach patients and demonstrate the benefits of treatment. The first step in achieving medication adherence should be educating patients about chronic diseases and serious complications associated with nonadherence. Education may also be valuable when the patient occasionally decides not to adhere because of a misunderstanding or scepticism over the use of the medication. Moreover, education is needed for self-management (for example, home BP monitoring) because it is one of the strategies for medication adherence and improved clinical outcomes.

Although education is an important factor, educational interventions alone represent a weak approach to enhance adherence to medication and lifestyle modifications. Patients need to be informed, encouraged and trained in the use of cognitive and behavioral self-regulation strategies. ${ }^{1}$ A multidisciplinary approach is needed to control chronic conditions and enhance patient adherence to prescribed pharmacotherapy, a cornerstone of successful disease treatment. ${ }^{2}$ This approach requires collaboration of a team of healthcare professionals working on different educational and behavioral strategies aimed at improving medication adherence. Effective management in chronic diseases can be potentially brought about by the combination of patient education, good physician-patient 
relationships and collaborative efforts from healthcare professionals to improve lifestyle modifications and medication adherence. To make this approach more effective, we believe that healthcare professionals, such as pharmacists, with their specific expertise and knowledge of medications, can have a very important role as patient educators and interventionists in various chronic conditions. Furthermore, pharmacists serve as an important link between patients and physicians. They are in a privileged position to convince patients about the importance of pharmacotherapy adherence in reducing overall healthcare expenditures and improving health outcomes. In the past two decades, pharmacists have been developing and implementing validated methods and services to enhance adherence to long-term medications and improve the clinical outcomes of chronic conditions. ${ }^{7}$

Almost all interventions that were effective for long-term treatment were complex, including combinations of more convenient care, information, reminders, self-monitoring, reinforcement, counselling, family therapy, psychological therapy, crisis intervention, manual telephone follow-up and supportive care. ${ }^{2}$

In the interesting study by Shin et $a l^{8}$, published in this issue of Hypertension Research, the authors compared hypertension management between cancer survivors and the general population, quantifying antihypertensive medication adherence, frequency of BP monitoring and perceived BP control in the two mentioned groups. The authors showed that cancer patients were significantly more likely to report full antihypertensive medication adherence and to monitor their BP. This group was also significantly more likely to perceive their BP as being very well controlled, compared with those in the comparison group. As most self-reported BP values are generally reliable and correlated with actual measured data, the authors assumed that hypertensive cancer patients are usually in better BP control than are hypertensive patients without cancer.

This research represents an addition to our knowledge of the factors influencing adherence to BP-lowering medication in hypertension. It points toward the hypothesis that cancer survivors have better practices regarding hypertension management compared with people without cancer. This difference is expected to be related to their different health perceptions and health behaviors, attributable to their personal cancer experiences. This effort by Shin et al. provides solid comments supporting the health belief model (value-expectancy theory), according to which the probability that an individual will take a preventive health action is a function of the perceived susceptibility to the disease, its severity, and the perceived benefits and barriers to the recommended preventive action. ${ }^{9}$ The expanded version of the health belief model also includes variables such as self-efficacy and cues to action, postulating that individuals' sense of self-efficacy and external cues (such as health education campaigns) also influence their health behavior (Figure 1). Despite several limitations well documented by authors in the 'Discussion' section, the information of this study is important and it did potentially add significant knowledge to the field, pointing to significant relationships between compliance and the health belief model theoretical variables. Although the authors did not determine whether cancer survivors have a perceived susceptibility specifically to hypertension, it can be speculated that cancer patients may have an increased perception of vulnerability with respect to their health, and this perceived vulnerability may lead to stricter medication adherence and BP monitoring. Shin et al. hypothesized that frequent BP monitoring and good adherence in cancer survivors could be a consequence of their increased concern for their health, rather than for the specific purpose of hypertension management. Indeed, adherence to long-term pharmacotherapy have traditionally been assumed to be better in cancer patients compared with other chronic diseases, due to the perceived understanding of the risks of not taking medication as prescribed. ${ }^{10}$

In many health care systems worldwide, oncological patients are incorporated into a multidisciplinary counselling plan that actively involves the patient coping with his problems and complaints by providing training, keeping protocols and assessing intervention successes. Thus, his self-responsibility is strengthened, and he gets the feeling that he is able to do something for himself to combat his disease. Investigations by the hospital for tumor biology in Freiburg (a cancer research center in Germany) have shown that patients with a greater sense of responsibility for themselves and a will to 'take their fate into their own hands' do have a higher life expectancy, becoming 'cancer survivors."

The study by Shin et al. has highlighted an important potential role for improved health education and follow-up measures to strengthen patients' perceptions about the benefits of treatment and compliance and patients' sense of self-efficacy. The next steps should be: (1) to use stronger experimental study designs to confirm the significance of the associative relationships found and (2) to pilot and implement cost-effective interventions to improve medication adherence and

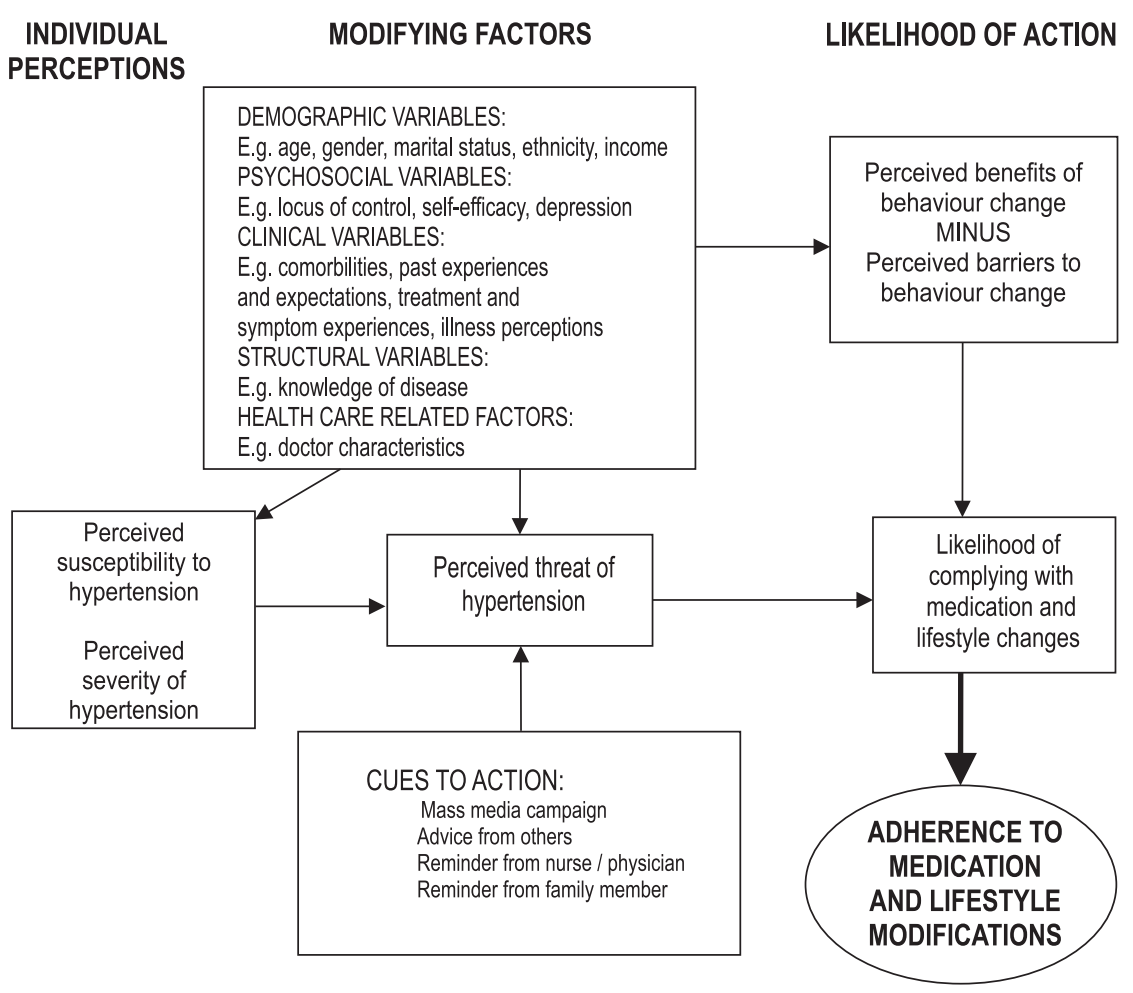

Figure 1 Expanded structure of the health belief model. 
associated outcomes in patients with chronic diseases.

\section{CONFLICT OF INTEREST}

The authors declare no conflict of interest.

1 Sabaté E. Adherence to Long-Term Therapies Evidence for Action. WHO, Geneva, Switzerland, 2003.

2 Haynes RB, Ackloo E, Sahota N, McDonald HP, Yao X. Interventions for enhancing medication adherence. Cochrane Database Syst Rev (Online) 2008: CD000011.
3 Sokol MC, McGuigan KA, Verbrugge RR, Epstein RS Impact of medication adherence on hospitalization risk and healthcare cost. Med Care 2005; 43: 521-530.

4 Pittman DG, Chen W, Bowlin SJ, Foody JM. Adherence to statins, subsequent healthcare costs, and cardiovascular hospitalizations. Am J Cardiol 2011; 107 : 1662-1666.

5 Osterberg L, Blaschke T. Adherence to medication. N Engl J Med 2005; 353: 487-497.

6 Krueger KP, Berger BA, Felkey B. Medication adherence and persistence: a comprehensive review. $A d v$ Ther 2005; 22: 313-356.

7 Hepler CD, Strand LM. Opportunities and responsibilities in pharmaceutical care. Am J Hospital Pharmacy 1990; 47: 533-543.
8 Shin DW, Kim SY, Cho J, Yang HK, Cho B Nam H-S, Kim H, Park J-H. Comparison of hypertension management between cancer survivors and the general public. Hypertens Res 2012; 35 935-939.

9 Rosenstock IM, Strecher VJ, Becker MH. Social learning theory and the health belief model. Health Educ Quarterly 1988; 15: 175-183.

10 Ruddy K, Mayer E, Partridge A. Patient adherence and persistence with oral anticancer treatment. CA Cancer J Clin 2009; 59: 56-66.

11 Eirmbter B. Preparing a counseling plan. In: Onkopress (ed). Quality Standard for the Oncology Pharmacy Service (quapos4), 4th edn. European Society of Oncology Pharmacy (ESOP), Oldenburg, 2008, pp 328-332. 